
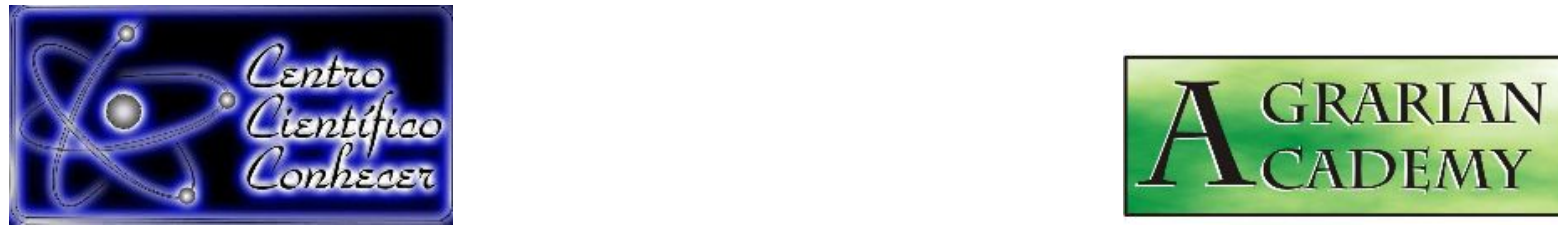

\title{
PRODUÇÃO E CRESCIMENTO INICIAL DA MORINGA EM DIFERENTES DOSES DE ESTERCO OVINO
}

\author{
Etiandro Djibany de Carvalho Simões Pereira ${ }^{1}$, Ciro de Miranda Pinto ${ }^{2}$, Maria Gorete \\ Flores Salles ${ }^{3}$, Olienaide Ribeiro de Oliveira Pinto ${ }^{4}$, Aderson Martins Viana Neto ${ }^{5}$ \\ ${ }^{1}$ Bachareal em Agronomia/ Universidade da Integração Internacional da Lusofonia \\ Afro-Brasileira (UNILAB), Redenção-CE, Brasil. \\ ${ }^{2}$ Professor Doutor da Universidade da Integração Internacional da Lusofonia Afro- \\ Brasileira (UNILAB), Redenção-CE, Brasil. E-mail: ciroagron@unilab.edu.br \\ ${ }^{3}$ Professora Doutora da Universidade da Integração Internacional da Lusofonia Afro- \\ Brasileira (UNILAB), Redenção-CE, Brasil. \\ ${ }^{4}$ Doutora, Bolsista do Programa Nacional de Pós-Doutorados/CAPES, Universidade \\ da Integração Internacional da Lusofonia Afro-Brasileira (UNILAB), Redenção-CE, Brasil. \\ ${ }^{5}$ Doutor em Zootecnia/ Universidade Federal do Ceará (UFC), Fortaleza-CE. Brasil.
}

Recebido em: 02/06/2019 - Aprovado em: 15/06/2019 - Publicado em: 22/07/2019 DOI: 10.18677/Agrarian_Academy_2019a27

\begin{abstract}
RESUMO
A moringa possui características de adaptação à região semiárida, como tolerância a salinidade e deficiência hídrica, crescendo e desenvolvendo-se em solos de baixa fertilidade, tendo várias utilizações como alimentação humana e animal, medicinal, purificação de água, controle de pragas, adubação verde, etc. Assim, o objetivo deste trabalho foi avaliar a melhor dose de esterco ovino na produção de mudas de moringa. Os tratamentos utilizados foram: $T_{1}$ : testemunha (zero grama de esterco ovino); $\mathrm{T}_{2}$ : 10 gramas de esterco ovino; $\mathrm{T}_{3}: 20$ gramas de esterco; $\mathrm{T}_{4}: 30$ gramas de esterco ovino; $\mathrm{T}_{5}$ : 40 gramas de esterco ovino e $\mathrm{T}_{6}: 50$ gramas de esterco ovino. Adotou-se o delineamento inteiramente casualizado com seis tratamentos e quatro repetições. As variáveis analisadas foram: diâmetro do caule (DC), altura da planta (AP), taxa de crescimento absoluto das plantas em altura (TCAAP), taxa de crescimento relativo das plantas em altura (TCRAP), taxa de crescimento absoluto do diâmetro do coleto das plantas (TCADC), taxa de crescimento relativo do diâmetro do coleto das plantas (TCRDC), matéria seca da parte aérea (MSPA), matéria seca da raiz (MSR), matéria seca (MST), volume de raiz (VR), MSR/MSPA e MSR/MST. As variáveis, altura de planta, diâmetro do coleto, taxa de crescimento absoluto de diâmetro do coleto e MSR/MST não apresentaram significância estatística em nenhum modelo de regressão polinomial. As variáveis TCAAP TCRAP, TCRDC e MSPA apresentaram ajustamento quadrático. A dose esterco ovina média ótima do experimento foi 25,10 grama saco $^{-1}$ considerando as variáveis TCAAP, TCRAP, TCRDC e MSPA.
\end{abstract}

PALAVRAS-CHAVE: Alometria, Adubação orgânica, Moringa oleifera Lam, Sustentabilidade ambiental, Semiárido. 


\title{
PRODUCTION AND INITIAL GROWTH OF MORINGA IN DIFFERENT DOSES OF MANURE SHEEP
}

\begin{abstract}
The moringa has characteristics of adaptation to the semi-arid region, such as tolerance to salinity and water deficiency, growing and developing in soils of low fertility, having several uses such as human and animal feeding, medicinal, water purification, pest control, green adubation, etc. Thus, the objective of this work was to evaluate the best dose of sheep manure in the production of moringa seedlings. The treatments used were: $\mathrm{T}_{1}$ : control (zero gram of sheep manure); $\mathrm{T}_{2}$ : 10 grams of sheep manure; $T_{3}: 20$ grams of manure; $T_{4}: 30$ grams of sheep manure; $T_{5}: 40$ grams of sheep manure and $T_{6}: 50$ grams of sheep manure. A completely randomized design with six treatments and four replications was adopted. The variables analyzed were stem diameter (DC), plant height (AP), absolute growth rate of plants at height (TCAAP), relative growth rate of plants at height (TCRAP), absolute growth rate of plant diameter (dry matter), root dry matter (DMW), dry matter (DMW), root volume (DM), dry matter (DMW), MSR / MSPA and MSR / MST. The variables, plant height, collection diameter, absolute growth rate of collection diameter and MSR / MST did not present statistical significance in any polynomial regression model. The TCAAP variables TCRAP, TCRDC and MSPA presented quadratic adjustment. The optimal

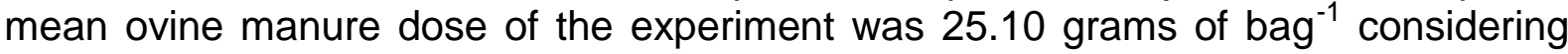
the variables TCAAP, TCRAP, TCRDC and MSPA.
\end{abstract}

KEYWORDS: Allometry, Organic fertilization, Moringa oleifera Lam, Sustainability Sustentabilidade, Semiarid.

\section{INTRODUÇÃO}

A Moringa oleifera Lamarck é uma planta nativa das partes ocidentais e subHimalaia do noroeste da Índia, Paquistão e Afeganistão. Essa planta também está distribuída e adaptada na região semiárida do nordeste do Brasil (SANTOS et., 2015). As características gerais são folhas compostas bipinadas, copa rala, de até $10 \mathrm{~m}$ de altura, flores esbranquiçadas, os frutos do tipo cápsula alada e deiscente com aspecto de uma vagem apresentando marcações por semente medindo até 35 cm (LORENZI; MATOS, 2008).

A planta de moringa é altamente valorizada, devido a sua composição nutricional, com alta gama de usos medicinais, sendo utilizadas no combate de avitaminoses $\mathrm{A}$ e $\mathrm{C}$, nos tratamentos de reumatismo e gota, como cicatrizantes de feridas, possuem diversos benefícios farmacológicos para o consumo humano, incluindo a promoção do crescimento, efeitos antimicrobianos, terapêuticos e antioxidantes (MUDGIL; BARAK, 2013).

Aplicações agronômicas da Moringa oleifera: extratos da moringa para controlar ácaro rajado, o Tetranychus urticae (HOLTZ et al., 2016), inseticida potencial para controlar os adultos de Sitophilus zeamais (caruncho) em grãos de milho armazenados (MATEUS et al., 2017), extratos no controle fungos como Fusarium solani e Rhizoctonia solani (GOSS et al., 2017), extratos foliar para avaliar a produção de pimentão, Capsicum annuum (MATTHEW, 2016), extrato foliar na produção de milho (WILLIAMs, et al., 2018), utilização como forragem na alimentação de caprinos (SULTANA et al., 2015), farinha de moringa no desempenho do crescimento de suínos (MUKUMBO et al., 2014).

As avaliações de análise de crescimento vegetal empregam características morfológicas e fisiológicas, quando as plantas são submetidas a diferentes tipos de 
tratamentos como: níveis de salinidade, doses de adubação aplicada no solo, doses de adubação foliar, concentração de $\mathrm{CO}_{2}$ via água de irrigação, doses de indutores de enraizamento, doses de fungos micorrizos etc. Essa técnica pode ser considerada um estudo destrutivo e não destrutivo dos vegetais. Cardoso et al., (2006) estudaram duas cultivares de algodão em diferentes arranjos de plantios, através da análise de crescimento não destrutiva como altura de planta, diâmetro do caulinar e comprimento da nervura principal da folha. Enquanto Nicolau (2018) estudou as respostas morfológicas e botânicas da moringa submetida à diferentes níveis de salinidade, para tanto empregou a análise de crescimento destrutiva e não destrutiva.

A adubação orgânica tem vantagens de melhorar as características físicas, químicas e microbiológicas de solos e em substratos. A matéria prima para adubação orgânica como estercos bovinos, ovinos e caprinos, a cama de frango dentre outros estão disponíveis nas propriedades agrícolas. A produção de mudas de moringa em diferentes substratos foi estudada por Neves et al. (2010) e Rodrigues et al., (2016). A adubação considerando diferentes fonte orgânicas na produção de mudas de moringa, esterco bovino, (SOUZA et al. 2015), a cama de frango por Souza et al. (2018), substrato comercial (ROSA et al. (2018). Nesse contexto, o objetivo desse trabalho foi produzir mudas de moringa sob diferentes doses de esterco ovino e acompanhar o desenvolvimento das plantas.

\section{Localização da área experimental}

\section{MATERIAL E MÉTODOS}

O material propagativo empregado no experimento em questão, as sementes da moringa foram coletadas no assentamento Nova assunção município de Aracoiaba, Ceará, nas coordenadas geográficas latitude (S) 4ำ 22' 16" longitude (WGr) 38 48' 51" nordeste (IPECE, 2017a).

O experimento foi conduzido na Universidade Internacional da Lusofonia AfroBrasileira (UNILAB), Campus das Auroras, localizado no município de Redenção, Ceará, nas coordenadas geográficas: latitude (S) 4 13' 33" e longitude (WGr) 38을 43' 50" nordeste (IPECE, 2017 b).

\section{Delineamento Experimental}

O delineamento estatístico adotado foi o inteiramente casualizado com 6 tratamentos e 4 repetições compostas de 4 sacos cada, totalizando 24 unidades experimentais e 96 sacos. Os tratamentos utilizados foram os seguintes: $T_{1}$ : testemunha (zero grama de esterco ovino); $\mathrm{T}_{2}: 10$ gramas de esterco ovino; $\mathrm{T}_{3}: 20$ gramas de esterco; $\mathrm{T}_{4}: 30$ gramas de esterco ovino; $\mathrm{T}_{5}: 40$ gramas de esterco ovino e $\mathrm{T}_{6}$ : 50 gramas de esterco ovino.

Para condução do experimento foram preparados os sacos de plástico do tamanho $20 \times 30 \mathrm{~cm}$ enchidos com arisco e adicionados diferentes doses de esterco ovino a cinco centímetros de profundidade. Foram semeadas duas sementes por saco numa profundidade de $3 \mathrm{~cm}, 10$ dias depois da emergência, realizou-se o desbaste, deixando apenas uma plântula a mais vigorosa. A instalação do experimento ocorreu no dia 21 de janeiro de 2019, a emergência de todas as plântulas ocorreu em 28 de janeiro de 2019. A primeira avaliação do experimento para altura de planta e diâmetro do coleto ocorreu no dia 5 de fevereiro de 2019. No dia 2 de maio de 2019 foi realizada a última tomada de dados de altura de planta, diâmetro do coleto. O experimento ocorreu durante 86 dias de avaliação. 


\section{Variáveis Analisadas}

As variáveis analisadas foram: diâmetro do caule (DC), altura da planta (AP), relação $A P / D C\left(\mathrm{~cm} \mathrm{~mm}^{-1}\right)$ e número de folhas (NF), a coleta de dados foi feita semanalmente no mesmo dia da semana, esses dados foram coletados e mensurados com ajuda de paquímetro digital para (DC) e trena para (AP). Os dados de altura e diâmetro do coleto foram avaliados também pelas taxas de crescimento absoluto e relativo adaptadas de Cairo et al. (2008), conforme equações 1 a 4:

$$
T C A A P=\frac{A P_{f}-A P_{i}}{T_{f}-T_{i}}
$$

TCAAP $=$ taxa de crescimento absoluto das plantas em altura $\left(\mathrm{cm} \mathrm{dia}^{-1}\right)$

$$
T C R A P=\frac{\operatorname{Ln}\left(A P_{f}\right)-\operatorname{Ln}\left(A P_{i}\right)}{T_{f}-T_{i}}
$$

TCRAP $=$ taxa de crescimento relativo das plantas em altura $\left(\mathrm{cm} \mathrm{cm}^{-1} \mathrm{dia}^{-1}\right)$;

$$
T C A D C=\frac{D C_{f}-D C_{\bar{i}}}{T_{f}-T_{\bar{i}}}
$$

TCADC $=$ taxa de crescimento absoluto do diâmetro do coleto das plantas $\left(\mathrm{mm} \mathrm{dia}^{-1}\right)$

$$
T C R D C=\frac{\operatorname{Ln}\left(D C_{f}\right)-\operatorname{Ln}\left(D C_{i}\right)}{T_{f}-T_{i}}
$$

TCRDC $=$ taxa de crescimento relativo do diâmetro do coleto das plantas $\left(\mathrm{mm} \mathrm{mm}^{-1}\right.$ $\left.\mathrm{dia}^{-1}\right)$;

$A P_{f}=$ altura das plantas ao final do experimento $(\mathrm{cm})$;

$A P_{i}=$ altura da planta no início do experimento $(\mathrm{cm})$;

$\mathrm{T}_{\mathrm{f}}=$ Tempo de avaliação final $(\mathrm{cm})$;

$T_{i}=$ Tempo de avaliação inicial $(\mathrm{cm})$;

As variáveis, matéria seca da parte aérea (MSPA), matéria seca da raiz (MSR), matéria seca (MST) foram obtidas após secagem em estufa numa temperatura de $72^{\circ} \mathrm{C}$ por 24 horas. Foram também obtidas as relações alométricas entre MSR/MSPA e MSR/MST. O volume de raiz foi obtido com proveta graduada, medindo a diferença de deslocamento entre o volume final (raiz + água) e volume inicial (água) na proveta. A análise de regressão polinomial foi estimada com auxilio do software estatístico ASSISTAT, versão 7.7 (SILVA; AZEVEDO, 2016).

\section{RESULTADOS E DISCUSSÃO}

O experimento foi conduzido sem interferência de nenhuma praga e doença. $\mathrm{Na}$ Tabela 1 constam os dados relativos à altura de planta e diâmetro do coleto da moringa em função das diferentes doses de esterco ovino, constatou-se na análise de variância que não ocorreu significância estatística em nenhum dos níveis da regressão polinomial $(p \geq 0,05)$. Como não ocorreu significância para regressão polinomial em altura de planta e diâmetro do coleto, essa variação pode ser em função de média e desvio de $45,76 \pm 3,34$ e $5,21 \pm 0,31$. O coeficiente de variação para a variável altura de planta e diâmetro do coleto foi igual a 7,30 e 5,95 \% 
respectivamente (Tabela 1). Ferreira (2018), afirma que $C V<10 \%$ é classificado como baixo, indicando ótima precisão experimental.

TABELA 1. Resumo da análise de variância para altura de planta (cm) e diâmetro do coleto $(\mathrm{mm})$ da moringa em função de doses de esterco ovino, Redenção, 2019.

\begin{tabular}{lcc}
\hline Fonte de variação & AP & DC \\
\cline { 2 - 3 } & Quadrado Médio & Quadrado Médio \\
\hline Reg.linear & $27,50022(\mathrm{p}=0,1339)$ & $0,25200(\mathrm{p}=0,1228)$ \\
Reg.quadrática & $40,39360(\mathrm{p}=0,0732)$ & $0,25054(\mathrm{p}=0,1239)$ \\
Reg.cúbica & $2,05868(\mathrm{p}=0,6726)$ & $0,01070(\mathrm{p}=0,7425)$ \\
Reg.4ㅇau & $5,58036(\mathrm{p}=0,4886)$ & $0,14108(\mathrm{p}=0,2415)$ \\
Reg.5-grau & $2,48016(\mathrm{p}=0,6430)$ & $0,00684(\mathrm{p}=0,7927)$ \\
\hline CV $(\%)$ & 7,30 & 5,95 \\
\hline
\end{tabular}

Para a variável altura de planta resultados semelhantes foram verificados por Rosa et al. (2018), estudando doses de fertilizante de liberação controlada, sendo: 0; 2; 4; 6 e $8 \mathrm{~kg} \mathrm{~m}^{-3}$ de substrato comercial para moringa. Resultados diferentes foram detectados por Souza et al. (2015) a altura da planta foi afetada positivamente com aumento da dosagem de esterco acrescentado no solo, a redução ocorreu a partir do ponto de máxima dose. Os mesmos autores afirmam que a quantidade de fertilizante orgânico na dose máxima foi suficiente para que as plantas de moringa expressassem o máximo de crescimento. Medeiros et al. (2017) constataram que a aplicação de diferentes volumes $(1: 0 ; 1: 1 ; 2: 1$; e $3: 1$, composto orgânico bovino para solo) de composto orgânico bovino proporcionou aumento na altura de mudas de moringa. Esse aumento ocorreu nos substratos que utilizaram 1:1; 2:1; e 3:1 em detrimento ao 1:0 (composto bovino sem solo).

No diâmetro do coleto da moringa os resultados foram não significativos ao nível de $5 \%$ de probabilidade em função das doses de esterco ovino (Tabela 1). Enquanto, Souza et al. (2015) estudando o crescimento e desenvolvimento da moringa em diferentes doses de esterco bovino estimaram um modelo quadrático para o diâmetro do colmo, tendo o coeficiente de determinação de 81,41 \%. Medeiros et al. (2017) descrevem o crescimento do diâmetro caulinar com aumento da dose de esterco bovino de modo satisfatório.

Souza et al. (2015) estudando o diâmetro de colmo da moringa, constataram redução com a elevação dos níveis de esterco bovino adicionados. O menor diâmetro foi obtido para as doses de $1.728,0$ e $3.456,0 \mathrm{~g} \mathrm{vaso}^{-1}$ de esterco, o que pode confirmar o mecanismo de adaptação desta espécie exótica às condições semiáridas do nordeste brasileiro. Rosa et al. (2018), estudando doses de fertilizante para o crescimento da moringa, estimaram o modelo quadrático $Y=5,2141+0,7586 x$ $0,0634 x^{2}(81,27 \%)$ para diâmetro do colmo, tendo dose ótima de $5,98 \mathrm{~kg} \mathrm{~m}^{-3}$.

A taxa de crescimento absoluto da altura (TCAAP) de planta de moringa para a regressão polinomial não apresentou significância estatística ao nível de $5 \%$ de probabilidade nas regressões de graus um, três, quatro e cinco, o grau dois foi significativo $(p=0,0200)$ na tabela 2 . A equação quadrática para TCAAP foi igual $Y=0,24362247+0.00530763 x-0.00008842 x^{2}$ com $R^{2} 94,63 \%$, tendo como dose ótima de esterco ovino $30,01 \mathrm{~g} \mathrm{saco}^{-1}$. A TCAAP na dose ótima de esterco ovino foi $0,3233 \mathrm{~cm} \mathrm{dia}^{-1}$.

$\mathrm{Na}$ Tabela 2, constam os dados da anova e decomposição dos graus de liberdade de tratamento nos respectivos graus de regressão polinomial para taxa de crescimento relativo da altura de planta da moringa (TCRAP). Não foi observada 
significância estatística ( $p \geq 0,05)$ nos modelos de regressão polinomial de grau um, três, quatro e cinco. Enquanto a regressão de segundo grau foi significativa a $5 \%$ de probabilidade. Os dados experimentais da taxa de crescimento relativo da altura de planta da moringa foram ajustados ao modelo quadrático 97,40\% (Tabela 2). A taxa de crescimento relativo da altura de planta máxima foi de $0,0013654 \mathrm{~cm} \mathrm{~cm}^{-1} \mathrm{dia}^{-1}$, valor calculado com base na dose ótima de $30,98 \mathrm{~g} \mathrm{saco}^{-1}$ de esterco ovino.

TABELA 2. Resumo da análise de variância e análise de regressão para taxa de crescimento absoluto da altura de planta -TCAAP da moringa $\left(\mathrm{cm} \mathrm{dia}^{-1}\right)$ e taxa de crescimento relativo da altura de planta - TCRAP $\left(\mathrm{cm} \mathrm{cm}^{-1} \mathrm{dia}^{-1}\right)$ em função de doses de esterco ovino, Redenção, 2019.

\begin{tabular}{lcc}
\hline Fonte de variação & TCAAP & TCRAP \\
\cline { 2 - 3 } & Quadrado Médio & Quadrado Médio \\
\hline Reg.linear & $0,00551(\mathrm{p}=0,0967)$ & $0,00000(\mathrm{p}=0,0501)$ \\
Reg.quadrática & $0,01167(\mathrm{p}=0,0200)$ & $0,00001(\mathrm{p}=0,0218)$ \\
Reg.cúbica & $0,00027(\mathrm{p}=0,7050)$ & $0,00000(\mathrm{p}=0,9164)$ \\
Reg.4ㅇau & $0,00020(\mathrm{p}=0,7427)$ & $0,00000(\mathrm{p}=0,9286)$ \\
Reg.5-grau & $0,00051(\mathrm{p}=0,6007)$ & $0,00000(\mathrm{p}=0,6123)$ \\
\hline CV(\%) & 14,35 & 5,95 \\
\hline Regressão TCAAP & $\mathrm{Y}=0,24362247+0.00530763 x-0.00008842 \mathrm{x}^{2}$ \\
& $\mathrm{R}^{2}=0,94638060($ Coeficiente de determinação) \\
\hline Regressão TCRAP & $\mathrm{Y}^{2}=0,00757299-0,00013358 x-0,000002156 \mathrm{x}^{2}$ \\
& $\mathrm{R}^{2}=0,97405643$ (Coeficiente de determinação) \\
\hline
\end{tabular}

A taxa de crescimento absoluto de diâmetro do coleto (TCADC) da moringa $\left(\mathrm{cm} \mathrm{dia}{ }^{-1}\right)$ não foi significativa $(p \geq 0,05)$ em todos os graus da regressão polinomial. $A$ equação para TCADC é expressa com $Y=0,03285 \pm 0,00701$ (Tabela 3). $A$ taxa de crescimento relativo de diâmetro do coleto (TCRDC) apresentou resposta significativa na regressão polinomial para os graus um $(p=0,0024)$, dois $(p=0,0035)$ e três $(p=0,0230)$ na tabela 3 . Nessa análise se considerada a equação de segundo grau $Y=0,01158015-0,00043946 x+0,00001611 x^{2}$, o coeficiente de variação é igual a 0,75 (Tabela 3). A dose ótima de esterco ovino foi 13,65 g por saco, a TCRDC foi $0,008583 \mathrm{~cm} \mathrm{~cm}^{-1} \mathrm{dia}^{-1}$. O coeficiente de variação da TCRDC foi 12,72 (Tabela 3).

TABELA 3. Resumo da análise de variância e análise de regressão para taxa de crescimento absoluto de diâmetro do coleto -TCADC da moringa $\left(\mathrm{cm} \mathrm{dia}^{-1}\right)$ e taxa de crescimento relativo de diâmetro do coleto - TCRDC $\left(\mathrm{cm} \mathrm{cm}^{-1} \mathrm{dia}^{-1}\right)$ em função de doses de esterco ovino, Redenção, 2019.

\begin{tabular}{|c|c|c|}
\hline \multirow[t]{2}{*}{ Fonte de variação } & TCADC & TCRDC \\
\hline & Quadrado Médio & Quadrado Médio \\
\hline Reg.linear & $0,00000(p=0,9516)$ & $0,00002(p=0,0024)$ \\
\hline Reg.quadrática & $0,00005(p=0,3724)$ & $0,00001(p=0,0035)$ \\
\hline Reg.cúbica & $0,00009(p=0,2268)$ & $0,00001(p=0,0230)$ \\
\hline Reg. $4^{\circ}$ grau & $0,00005(p=0,3683)$ & $0,00000(p=0,2519)$ \\
\hline Reg.5grau & $0,00003(p=0,4808)$ & $0,00000(p=0,6735)$ \\
\hline $\mathrm{CV}(\%)$ & 22,53 & 12,72 \\
\hline Regressão TCADC & \multicolumn{2}{|c|}{$Y=0,03285 \pm 0,00701$} \\
\hline Regressão TCRDC & \multicolumn{2}{|c|}{$\begin{array}{l}Y=0,01158015-0,00043946 x-0,00001611 x^{2}-0.00000017 x^{3} \\
R^{2}=0,94949843 \text { (Coeficiente de determinação) }\end{array}$} \\
\hline
\end{tabular}


A matéria seca da parte aérea (MSPA) teve ajustamento ao modelo polinomial quadrático com ponto de máxima $(p=0,0087)$ na tabela 4 . A dose ótima foi $25,75 \mathrm{~g}$ de esterco ovino saco $^{-1}$, proporcionando um valor de MSPA de 3,01 g. A MSPA na dose ótima foi $3,01 \mathrm{~g}$, isso corresponde um aumento de $47,53 \%$ percentual em relação à dose zero (testemunha). O coeficiente de determinação foi 0,8110 (Tabela 4), expressa que o modelo quadrado é ajustado aos dados experimentais com $81,10 \%$, tendo uma variação não aplicada de $18,90 \%$. Rosa et al., (2018) pesquisando o crescimento da moringa em função de doses do substrato comercial, estimaram modelo quadrático $Y=22,1718+6,5780 x-0,5093 x^{2}$ e $R^{2}=0,94$ para MSPA. Souza et al. (2015), estudando diferentes doses de esterco bovino na produção de mudas de moringa, estimaram um modelo quadrático com $R^{2}=0,70$. Nessa equação o valor máximo de MSPA foi obtido na dose $432 \mathrm{~g} \mathrm{vaso}^{-1}$.

A matéria seca da raiz (MSR) não apresentou ajustamento polinomial aos modelos linear, quadrática e quártico. O ajustamento ocorreu nos modelos de regressão de grau três $(p=0,0309)$ e cinco $(p=0,0017)$ com significância estatística (Tabela 4). O coeficiente de variação foi $13,49 \%$. O coeficiente de determinação $99,99 \%$, expressa o ajustamento ao modelo de grau cinco. Ausência de resposta na matéria seca de raiz, em função da aplicação de doses de substrato no crescimento da moringa foi verificado por Rosa et al., (2018). Souza et al. (2018) estudaram o crescimento da moringa, e constataram redução na matéria seca de raiz com o avançar das doses de cama de frango. Rodrigues et al., (2016) verificaram que matéria seca de raiz de moringa, nas duas proporções maiores de proporções de composto orgânico em relação a fibra de coco verde.

TABELA 4. Resumo da análise de variância e análise de regressão para matéria seca da parte aérea (MSPA, g) e matéria seca da raiz (MSR, g) em função de doses de esterco ovino, Redenção, 2019.

\begin{tabular}{|c|c|c|}
\hline \multirow[t]{2}{*}{ Fonte de variação } & MSPA & MSR \\
\hline & Quadrado Médio & Quadrado Médio \\
\hline Reg.linear & $0,02657(p=0,7643)$ & $0,20997(p=0,6536)$ \\
\hline Reg.quadrática & $2,48660(p=0,0087)$ & $3,75550(\mathrm{p}=0,0695)$ \\
\hline Reg.cúbica & $0,32258(\mathrm{p}=0,303)$ & $5,52870(p=0,0309)$ \\
\hline Reg.4ํgrau & $0,25413(\mathrm{p}=0,3591)$ & $0,63113(\mathrm{p}=0,4392)$ \\
\hline Reg.5grau & $0,00893(\mathrm{p}=0,8618)$ & $13,53455(\mathrm{p}=0,0017)$ \\
\hline $\mathrm{CV}(\%)$ & 20,33 & 13,49 \\
\hline Regressão - MSPA & \multicolumn{2}{|c|}{$\begin{array}{l}Y=2,15647321+0.06646830 x-0,00129040 x^{2} \\
R^{2}=0,81101265 \text { (Coeficiente de determinação) }\end{array}$} \\
\hline Regressão - MSR & \multicolumn{2}{|c|}{$\begin{array}{l}0,01295659 x^{3}-0,00029979 x^{4}+0,00000243 x^{5} \\
R^{2}=0,99999998 \text { (Coeficiente de determinação) }\end{array}$} \\
\hline
\end{tabular}

A matéria seca (MST) e volume de raiz (VR) apresentaram ajustamento ao modelo polinomial de grau cinco, tendo um coeficiente de determinação igual a 99,99\%. O coeficiente de variação para MST e VR foi 11,20 e 1,97\%, respectivamente (Tabela 5). Rodrigues et al., (2016) estudaram a produção de moringa e constataram aumento na matéria seca total das plântulas nas proporções 75:25 e 100:00 de composto orgânico: fibra de coco verde, tendo coeficiente de variação de 31\%. Em estudo realizado por Rosa et al., (2018) com a cultura da 
moringa em função de doses do substrato comercial, o volume de raízes não apresentou alterações significativas.

TABELA 5. Resumo da análise de variância e análise de regressão para matéria seca total (MST, g) e volume de raiz (VR, $\mathrm{mL}$ ) em função de doses de esterco ovino, Redenção, 2019.

\begin{tabular}{|c|c|c|}
\hline \multirow[t]{2}{*}{ Fonte de variação } & MST & VR \\
\hline & Quadrado Médio & Quadrado Médio \\
\hline $\begin{array}{l}\text { Reg.linear } \\
\text { Reg.quadrática } \\
\text { Reg.cúbica } \\
\text { Reg.4ํgrau } \\
\text { Reg.5ํa }\end{array}$ & $\begin{array}{r}0,38591(p=0,5886) \\
12,35388(p=0,0059) \\
8,52220(p=0,0186) \\
1,68622(p=0,2648) \\
12,84822(p=0,0052)\end{array}$ & $\begin{array}{r}15,43951(p=0,0007) \\
4,01953(p=0,053) \\
4,71259(p=0,0378) \\
0,02734(p=0,8662) \\
11,94947(p=0,0021)\end{array}$ \\
\hline $\mathrm{CV}(\%)$ & 11,20 & 1,97 \\
\hline Regressão - MSPA & \multicolumn{2}{|c|}{$\begin{array}{l}Y=8,40062500+1,51682500 x-0,22170156 x^{2}+0,01237372 \\
x^{3}-0,00028920 x^{4}+0,00000237 x^{5} \\
R^{2}=0,99999998 \text { (Coeficiente de determinação) }\end{array}$} \\
\hline Regressão - MSR & $\begin{array}{l}Y=47,06250000+1.5 \\
0,01249219 x^{3}+0,00028 \\
R^{2}=0,99999998 \text { (Coefici }\end{array}$ & $\begin{array}{l}67 x-\quad 0,22807292 \\
0,00000229 x^{5} \\
\text { e determinação) }\end{array}$ \\
\hline
\end{tabular}

A relação alométrica entre MSR/MSPA foi significativa $(p=0,0311)$ apenas ao modelo de regressão de grau cinco, que teve um coeficiente de determinação igual $99,99 \%$. O coeficiente de variação foi igual $23,13 \%$, expressando uma dispersão do desvio padrão em torno da média aritmética (Tabela 6). A relação entre MSR/MSPA foi estudada por Souza et al. (2018) que constataram incremento linear $Y=1,8+0,0344 x$ e $R^{2}=86,11 \%$, nas doses de cama de frango $0,40,80$ e $120 \mathrm{~g} \mathrm{dm}^{-3}$, denotando que o acúmulo de matéria de raiz foi influenciado positivamente pelo composto orgânico e consequente para parte área da plântula de moringa.

TABELA 6. Relação MSR/MSPA e MSR/MST em função de doses de esterco ovino, Redenção, 2019.

\begin{tabular}{|c|c|c|}
\hline \multirow[t]{2}{*}{ Fonte de variação } & MSR/MSPA & MSR/MST \\
\hline & Quadrado Médio & Quadrado Médio \\
\hline Reg.linear & $0,00119(p=0,9597)$ & $0,00001(p=0,9330)$ \\
\hline Reg.quadrática & $0,55214(\mathrm{p}=0,2863)$ & $0,00493(\mathrm{p}=0,1214)$ \\
\hline Reg.cúbica & $0,16888(p=0,5510)$ & $0,00088(p=0,5015)$ \\
\hline Reg.4ํgrau & $0,00150(p=0,9548)$ & $0,00018(\mathrm{p}=0,7590)$ \\
\hline Reg.5grau & $2,50081(\mathrm{p}=0,0311)$ & $0,00802(\mathrm{p}=0,0526)$ \\
\hline $\mathrm{CV}(\%)$ & \multicolumn{2}{|l|}{23,13} \\
\hline $\begin{array}{l}\text { Regressão } \\
\text { MSPA/MST }\end{array}$ & \multicolumn{2}{|c|}{$\begin{array}{l}Y=3,01516000+0,58116298 \quad x-0,10089257 \quad x^{2}+ \\
0,00569906 x^{3}-0,00013054 x^{4}+0,00000105 x^{5} \\
R^{2}=0,99999998 \text { (Coeficiente de determinação) }\end{array}$} \\
\hline $\begin{array}{l}\text { Regressão } \\
\text { MSR/MSTT }\end{array}$ & \multicolumn{2}{|c|}{$Y=0,73758 \pm 0,04324$} \\
\hline
\end{tabular}

A MSR/MST não apresentou significância a nenhum modelo polinomial $(p \geq 0,05)$, teve um coeficiente de variação de $5,86 \%$. Nesse caso a variação dos AGRARIAN ACADEMY, Centro Científico Conhecer - Goiânia, v.6, n.11; p. 288 
dados pode ser expressa como uma função de média e desvio padrão, Y MSR/MST $=0,73758 \pm 0,04324$ (Tabela 6).

\section{CONCLUSÃO}

A partir da produção das mudas de moringa em diferentes doses de esterco ovino e após acompanhar desenvolvimento da planta, a dose de esterco ovina média ótima do experimento é 25,10 grama saco ${ }^{-1}$ considerando as variáveis taxa de crescimento relativo da altura, taxa de crescimento absoluto da altura de altura, taxa de crescimento relativo de diâmetro do coleto e matéria seca da parte aérea.

\section{REFERÊNCIAS}

CAIRO, P.A.R.; OLIVEIRA, L.E.M.; MESQUITA, A.C. Análise de crescimento de plantas. Vitória da Conquista. Edições. UESB, 2008,72p.

CARDOSO, G. D.; ALVES, P. L. C.A ; BELTRÃO, N. E. M.; BARRETO, A. F. Uso da Análise de crescimento não destrutiva como ferramenta para avaliação de cultivares. Revista de Biologia e Ciências da Terra, v.6, n. 2, 2ํㅗㄴ semestre, p.79-84, 2006. Disponível em: <http://joaootavio.com.br/bioterra/workspace/uploads/artigos/algodoeiro5181a66452181.pdf>.

FERREIRA, P. V. Estatística Experimental Aplicada às Ciências Agrárias. Editora UFV, Edição1ạ, 2018, 588p.

IPCE. (2017, a). Instituto de Pesquisa e Estratégia Econômica do Ceará. Perfil básico municipal - Aracoiaba. Fortaleza, Ceará. Disponível em: $<$ https://www.ipece.ce.gov.br/wpcontent/uploads/sites/45/2018/09/Aracoiaba_2017.p df>.

IPCE. (2017, b). Instituto de Pesquisa e Estratégia Econômica do Ceará. Perfil básico municipal - Redenção. Fortaleza, Ceará. Disponível em: $<$ https://www.ipece.ce.gov.br/wpcontent/uploads/sites/45/2018/09/Redencao_2017.p $\mathrm{df}>$.

GOSS, M.; MAFONGOYA, P.; GUBBA, A. Moringa oleifera extracts effect on Fusarium solani and Rhizoctonia solani growth. Asian Research Journal of Agriculture, v.6, n.1, p1-10, 2017. Disponível em: <http://www.journalarja.com/index.php/ARJA/article/view/1714/2762>.

HOLTZ, A. M.; CARVALHO, J. R.; FRANZIN, M. F.; PIRES, A. A.; THAIS COFFLER, T.; JOHNATAN MARCHIORI, J. J. P. Toxicidade de extratos aquosos de Moringa oleifera para Tetranychus urticae. Revista Ifes Ciência, v.1, n 3, p.4-13, 2016, Instituto Federal do Espírito Santo. Disponível em: http://ojs.ifes.edu.br/index.php/ric/article/view/502/399.

LORENZI, H.; MATOS, F.J.A. Plantas medicinais no Brasil: nativas e exóticas. 2.ed. Nova Odessa: Instituto Plantarum, 2008, 544p.

MAteus, A. E.; AZEVEDO, F. R.; ALVeS, A. C. L.; FeItOSA, J. V. Potencial da Moringa oleifera como inseticida no controle de adultos de Sitophilus zeamais 
(Coleoptera: Curculionidae) em grãos de milho armazenados. Acta Iguazu, Cascavel, v.6, n.2, p. 112-122, 2017. Disponível em: http://erevista.unioeste.br/index.php/actaiguazu/article/view/17538/11687.

MATTHEW, A. Moringa leaf extract on the growth and yield of pepper (Capsicum annuum L.). ARPN Journal of Agricultural and Biological Science, v.11, n. 3, 2016.

Disponível

em: http://www.arpnjournals.org/jabs/research_papers/rp_2016/jabs_0316_785.pdf.

MEDEIROS, R. L. S.; CAVALCANTE, A. G..; CAVALCANTE, A. C. P. SOUZA, V. C. Crescimento e qualidade de mudas de Moringa oleifera lam em diferentes proporções de composto orgânico. Revista Ifes Ciência, Instituto Federal do Espírito Santo, v. 3, n.1, p.204-216, 2017. Disponível em: $<$ http://ojs.ifes.edu.br/index.php/ric/article/view/777/487>.

MUDGIL, D.; BARAK, S. Composition, properties and health benefits of indigestible carbohydrate polymers as dietary fiber: A review. International Journal of Biological Macromolecules, n.61, p.1-6, 2013. DOI:10.1016/j.ijbiomac.2013.06.044.

MUKUMBO, F. E.; MAPHOSA, V.; HUGO, A.; NKUKWANA, T. T.; MABUSELA, T. P.; MUCHENJE, V. Effect of Moringa oleifera leaf meal on finisher pig growth performance, meat quality, shelf life and fatty acid composition of pork. South African Journal of Animal Science, v.44, n.4, P. 388-400, 2014. Disponível em: $<$ https://www.ajol.info/index.php/sajas/article/view/111153/100922>. DOI: http://dx.doi.org/10.4314/sajas.v44i4.9.

NEVES, J. M. G.; SILVA, H. P.; DUARTE, R. F. Uso de substratos alternativos para produção de mudas de moringas. Revista Verde de Agroecologia e Desenvolvimento Sustentável, Mossoró - RN - Brasil, v.5, n.1, p.173 - 177, janeiro/março, $2010 . \quad$ Disponível em: <https://www.gvaa.com.br/revista/index.php/RVADS/article/view/259/259>.

NICOLAU, J. P.B. Qualidade de mudas de moringa (Moringa oleifera Lam.) Irrigadas com águas salinas. 2018, 28f. Trabalho de Conclusão de Curso de Especialização de Curso (Especialização- Gestão em Recursos Ambientais do Semiárido)- Instituto Federal de Educação Tecnológica da Paraíba, Picuí, IFPB, $2018 . \quad$ Disponível em: $<$ http://repositorio.ifpb.edu.br/jspui/bitstream/177683/396/1/25\%20\%20TCC\%202018 \%20-\%20Josefa\%20Patricia.pdf>.

RODRIGUES, L. A.; MUNIZ, T. A.; SAMARÃO, S. S.; CYRINO, A. E. Qualidade de mudas de Moringa oleifera Lam. Cultivadas em substratos com fibra de coco verde e compostos orgânicos. Revista Ceres, v. 63, n.4, p. 545-552, jul/ago, 2016. Disponível em: <http://www.ceres.ufv.br/ojs/index.php/ceres/article/view/1755/2248>.

ROSA, T. L. M.; JORDAIM, R. B.; ALEXANDRE, R. S.; ARAUJO, C. P.; GONÇALVES, F. G.; LOPES, J. C. Controlled release fertilizer in the growth of Moringa oleifera Lam. seedlings. FLORESTA, Curitiba, PR, v. 48, n.3. p. 303- 310, 
Julho/Setembro,

2018.

Disponível

em:

https://revistas.ufpr.br/floresta/article/view/50063/35484.

SANTOS, A. F. S; LUZ, L. A; PONTUAL, E. V.; NAPOLEÃO, T. H.; PAIVA, P. M.G.; COELHO, L. C. B. B. Moringa oleifera: Resource Management and Multiuse Life Tree. Advances in Research, v. 4, n.6, p.388-402, 2015. Disponível em : http://www.journalrepository.org/media/journals/AIR_31/2015/May/Coelho462015AIR 18177.pdf. DOI : 10.9734/AIR/2015/18177.

SILVA, F. A.S.; AZEVEDO, A.V. The Assistat Software Version 7.7 and its use in the analysis of experimental data. African Journal of Agricultural Research, v11, n.39, p. 3733-3740, 2016. Disponível em: https://academicjournals.org/journal/AJAR/article-full-text-pdf/5E8596460818.

SOUZA, F. M.; PEREIRA, W. E.; DANTAS, J. S.; NÓBREGA, J. S.; LIMA, E. C. S.; SÁ, F. V. S. Initial growth of Moringa oleifera Lam. as a function of poultry litter doses and granulometry. Pesquisa Agropecuária Tropical, v. 48, n. 4, p. 399-406, 2018. Disponível em: <https://www.revistas.ufg.br/pat/article/view/52447/26636>.

SOUZA, T. M. A.; SOUSA, T.A.; OLIVEIRA NETO, H. T.; SOUTO, L. S.; DUTRA FILHO, J. A.; MEDEIROS, A. C. Crescimento e desenvolvimento inicial da cultura da moringa (Moringa oleifera Lam.) submetida à fertilização orgânica. Revista Verde de Agroecologia e Desenvolvimento Sustentável, Pombal, Paraíba, v.10, n.5 (ESPECIAL), p.103-107, Dezembro, 2015. Disponível em: <https://www.gvaa.com.br/revista/index.php/RVADS/article/view/4268/3727. DOI: http://dx.doi.org/10.18378/rvads.v10i5.4268>.

SULTANA, N.; ALIMON, A. R.; HUQUE, K.S.; M. BABA' M.; HOSSAIN, J. Evaluation of Moringa Foliage (Moringa oleifera) as Goat Feed. Iranian Journal of Applied Animal Science, v. 5, n.4, p.865-871, 2015. Disponível em: <http://ijas.iaurasht.ac.ir/article_516457_0071e1bb2e751cdb4a0b7c81f6f88ac2.pdf>.

WILLIAMS, O.A.; OGUNWANDE, O.A.; AMAO, A.O. potentials of moringa oleifera leaf extract in increasing maize (Zea mays L.) productivity in Nigeria. International Journal of Scientific and Research Publications, v.8, n. 12, p. 279-290, 2018. Disponível em : http://www.ijsrp.org/research-paper-1218.php?rp=P848028. http://dx.doi.org/10.29322//JSRP.8.12.2018.p8438. 